\title{
Multiple Supplemental and Impacted Teeth (Polydent): A Non- Syndromic Case Report
}

\author{
Louis ZG Touyz ${ }^{*}$, Daphna Vermes ${ }^{2}$ \\ ${ }^{1}$ McGill University Faculty of Dentistry, PQ Canada \\ ${ }^{2}$ Seaforth Medical Center, Montreal, PQ Canada \\ *Corresponding Author: Louis ZG Touyz, Director and Professor Periodontics, McGill University Faculty of \\ Dentistry, PQ Canada; Tel: +5149317451; Email: Touyzlouis@gmail.com \\ Received Date: 01-06-2020; Accepted Date: 30-06-2020; Published Date: 07-07-2020 \\ Copyright $^{\circledR} 2020$ by Touyz LZG, et al. All rights reserved. This is an open access article distributed under the \\ terms of the Creative Commons Attribution License, which permits unrestricted use, distribution, and \\ reproduction in any medium, provided the original author and source are credited.
}

\begin{abstract}
Many cases of supernumerary teeth are found in congenital syndromes. Most prevalent dental modifications found, are the number of teeth in genetic conditions affecting ectodermal structures including Cleidocranial Dysostosis (CCD), hypohydrotic ectodermal dysplasia, focal dermal hypoplasia, craniofacial dysostosis, and aperts syndrome. The patient's main complaint was of toothache. Clinically there were no macroscopically detectable abnormalities, and he was unaware of any dental problems. His physical and facial appearance showed no deviations from normal, and he chewed, swallowed and spoke without impediment. A complement of permanent human teeth is usually 32 , within four quadrants of the mouth; from the center point there are two incisors, one canine, two pre-molars and three molars. Total $4 \times 8=32$ teeth and humans rarely develop more. Presented here is a case with multiple extra supplemental teeth eight in number in his permanent dentition, most of which six were identified radiographically and impacted. This case presented no other identifiable abnormalities in the mouth. The patient's pain derived from one supplemental upper premolar, which was removed. Exodontia other teeth not in function was advised, the patient acknowledged this but failed to return. This unique presentation with supplemental teeth and impactions was deemed to be non-syndromic.
\end{abstract}

\section{Keywords}

Concrescence; Gemination; Polydent; Supplemental-teeth

Touyz LZG | Volume 1; Issue 2 (2020) | JDHOR-1(2)-009 | Case Report

Citation: Touyz LZG. Multiple Supplemental and Impacted Teeth (Polydent): A Non-Syndromic Case

Report. J Dental Health Oral Res. 2020;1(2):1-8.

DOI: http://dx.doi.org/10.46889/JDHOR.2020.1204 


\section{Introduction}

Historically human teeth have been the subject of study for a long time by morphologist, comparative anatomists, palaeontologists, biologists, doctors and dentists. Humans are diphyodont (have 2 sets of teeth, deciduous and permanent) and are polymorphodont (have different shaped teeth, incisors, canines, premolars and molars). The embryonic stages of odontogenesis occurs early during pregnancy (starting 14 weeks in utero) and after birth (until about 20 years) $[1,2]$. During the initial ectodermal growth and proliferation of the face and cranium, a stoma forms; then an intraoral dental lamina forms in both jaws of the stoma. For every tooth, a dental bud develops an individual follicle on the dental lamina, and each goes through stages of being a histological cap, a bud and bell. From this a tooth forming organelle, an inner and outer ectodermal epithelium encases a mesodermal dental papilla. Enamel grows from the inner dental epithelium and induces subsequent formation of dentine in the underlying mesodermal dental papilla. An enamel crown, with the dentinal body forming the rest of the tooth, root, pulp and periodontal ligament, grows to form each tooth unit [2]. The nature of controlling mechanisms involved, which coordinate the expression of regulatory genes, remains obscure. Yet epigenetic cell dependant interactions are known to exist, when two tissues of different progeny and properties (mesoderm, mesoderm and/or ectoderm) become intimately juxtapositioned an alteration of the developmental course of the interactants manifest [3]. Human deciduous teeth start erupting after six months, and all erupt by age 10 years. The number of deciduous teeth in each quadrant is: two Incisors ( I ), one Canine ( C ), and two Molars $(\mathrm{M})$ : this amounts to $2-\mathrm{I}+1-\mathrm{C}+2-\mathrm{M}=5$ deciduous teeth in each quadrant; yielding 5 D-teeth $\times 4$ Quadrants $=20$ deciduous teeth. A full complement of permanent human teeth is usually 32 , namely in four quadrants of the mouth, from the center point there will be two incisors (2-I), one canine (1-C) two pre-molars (2-PM) and three molars (3-M) (2-I, 1-C, 2-PM, $3-\mathrm{M}=8$ per quadrant; $\times 4=32$ teeth) $[2,4,5]$. Missing or extra teeth are frequently found in genetic disorders involving ectoderm [6-8]. Additional teeth are relatively common. The most frequent permanent extra teeth which grow are extra incisors followed by molars (usually a fourth molar or more), then premolars and lastly extra canines [9]. When extra teeth are simple conical shaped, they are called 'supernumerary', but when they resemble morphology in a regular dentition, they are called 'supplemental teeth' [10]. When the size or number of teeth grow (collectively referred to as Tooth-Material: TM) exceeds the oral space available and cannot accommodate the erupting crowns (the TM), the teeth become stuck in the bone, and are referred to as "impactions in bone" [5]. Sometimes supplemental teeth are unusually impacted or disoriented, lying at an angle horizontally or totally inverted; this may affect other teeth in the arch too [11]. Non syndromic multiple supernumerary and supplemental teeth occur frequently in families, and is influenced by inherited traits [12]. Malformations of teeth exist when roots or crowns fuse; concrescence has one crown on one or more roots and germination

Touyz LZG | Volume 1; Issue 2 (2020) | JDHOR-1(2)-009 | Case Report

Citation: Touyz LZG. Multiple Supplemental and Impacted Teeth (Polydent): A Non-Syndromic Case Report. J Dental Health Oral Res. 2020;1(2):1-8.

DOI: http://dx.doi.org/10.46889/JDHOR.2020.1204 
occurs when there is two or more distinguishable crowns on one root. Germination is an autosomal sexually (female) linked dominant gene [13].

\section{Aim}

This report records an unusual case of a man unaware of his condition, who presented clinically with multiple supplemental teeth, (without any recognizable syndrome) and whose radiographs revealed multiple impactions and the full extent of his condition.

\section{Clinical Presentation}

A 35 year old man of color presented at a dental clinic complaining of ill-defined tooth ache in his left upper jaw. He had never sought help from any medical health professionals as he never had any problems, enjoyed good health, and was unaware of any dental trouble. His medical history revealed nothing unusual, and there was no inherited or genetic family history of any tooth related troubles.

This man presented for a dentally sourced problem to a private for-profit general dental practitioners clinic; he appeared healthy, functional, lucid and in excellent overall health. Besides the intra-oral exam which revealed the dental abnormalities described, the examination included an extra-oral exam, which revealed nothing untoward or problematic.

On examination supplemental premolars were noticed in position 35 and 45 (Fig. 1-3).

On Radiography eight supplemental teeth were seen (Fig. 4 and 5).

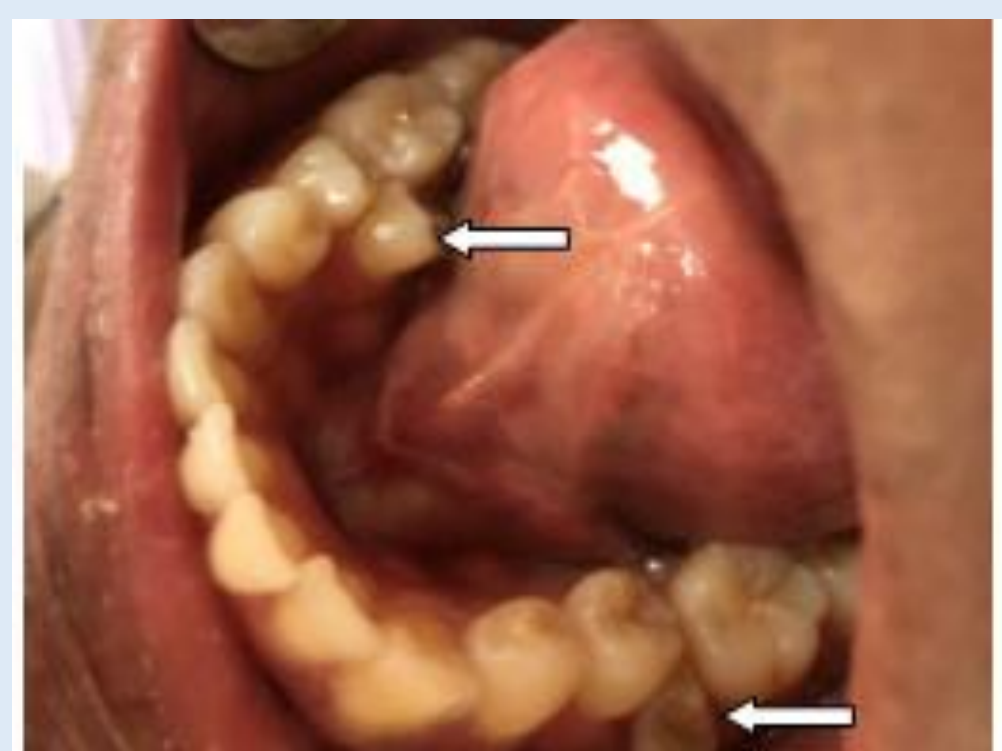

Figure 1: Lower arch. Supplemental crowns of premolar (arrows) in position \#35 and \# 45.

Touyz LZG | Volume 1; Issue 2 (2020) | JDHOR-1(2)-009 | Case Report

Citation: Touyz LZG. Multiple Supplemental and Impacted Teeth (Polydent): A Non-Syndromic Case Report. J Dental Health Oral Res. 2020;1(2):1-8. 


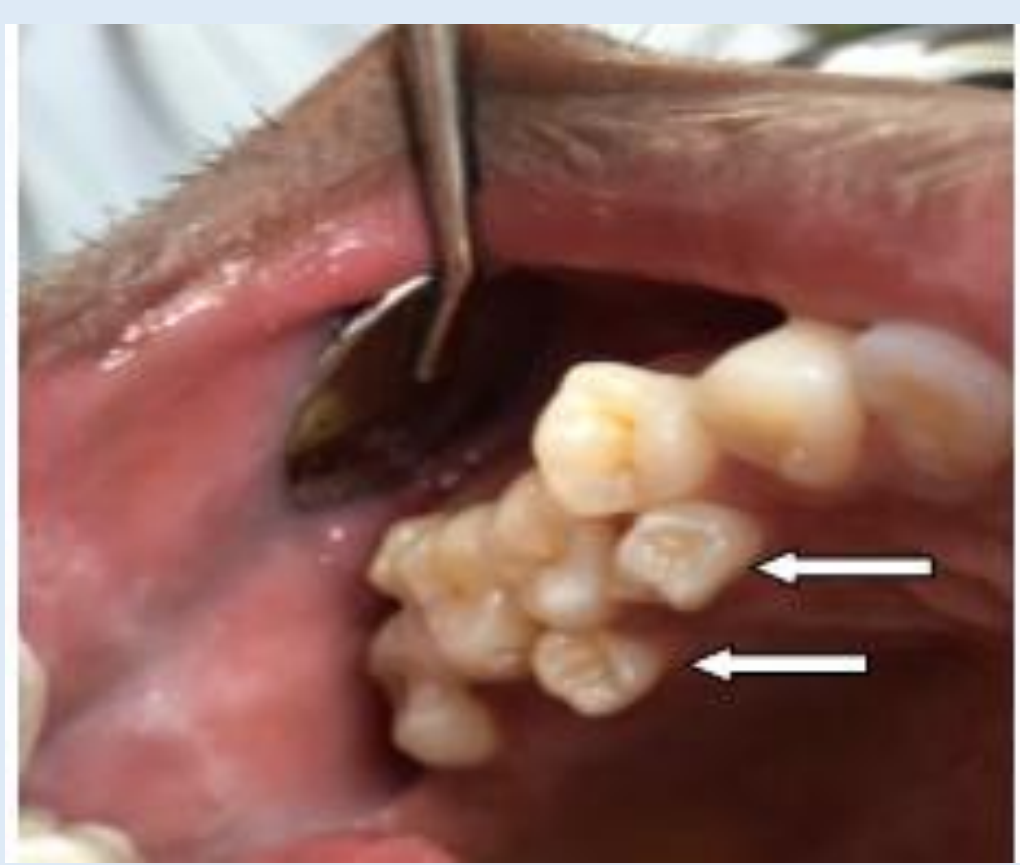

Figure 2: Upper right maxilla. Note supplemental premolars in the palate opposite \#14 and $\# 15$.

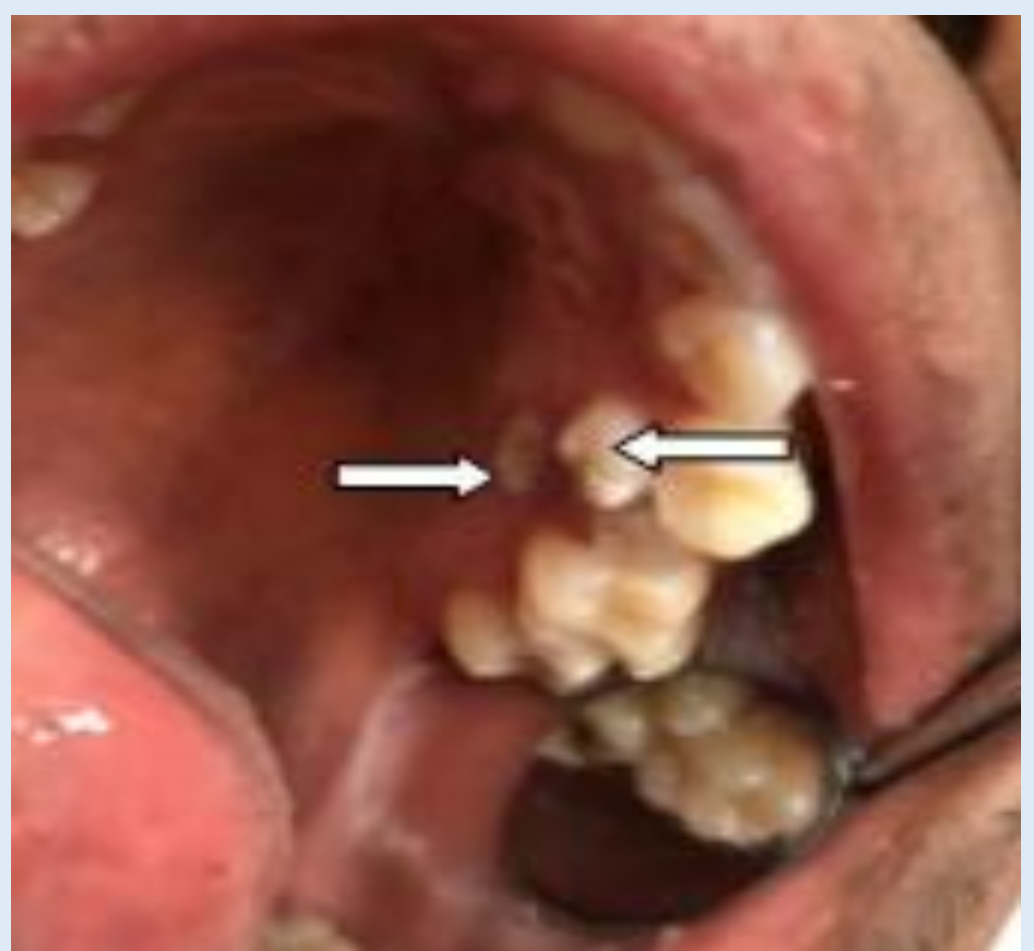

Figure 3: Upper left maxilla: Note two supplemental premolar in the palate opposite \#24.

The extra fully formed horizontal supplemental impacted premolar \#35 on the lower left mandible. The lower third molars (teeth \#38 and \#48) are impacted. There is a molar tooth \#18,

Touyz LZG | Volume 1; Issue 2 (2020) | JDHOR-1(2)-009 | Case Report

Citation: Touyz LZG. Multiple Supplemental and Impacted Teeth (Polydent): A Non-Syndromic Case Report. J Dental Health Oral Res. 2020;1(2):1-8. 
and supplemental molar tooth \#19 and as well a supplemental pre-molar \#14 and supplemental pre-molar \#15; there is a molar tooth \#28 and a supplemental molar \#29, as well as 2 supplemental pre-molar teeth (an extra \#24 and an extra \#25.) In total there are eight supplemental teeth (Fig. 5).

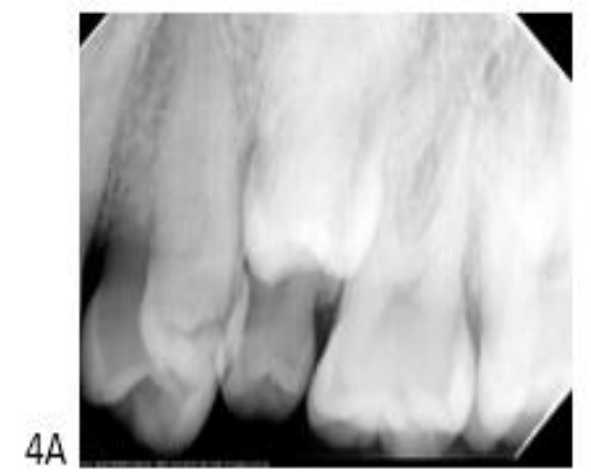

$4 B$

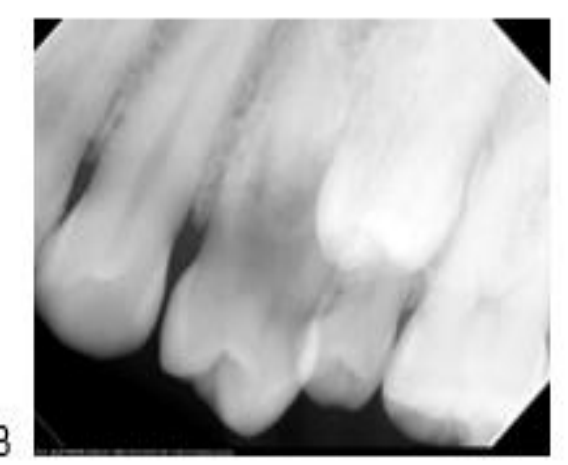

Figure 4: Intra-oral Radiographs. 4A and $4 \mathrm{~B}$ shows fully formed supplemental premolars. 4B the second supplemental premolar is not clear but discernible over the root of \#24.

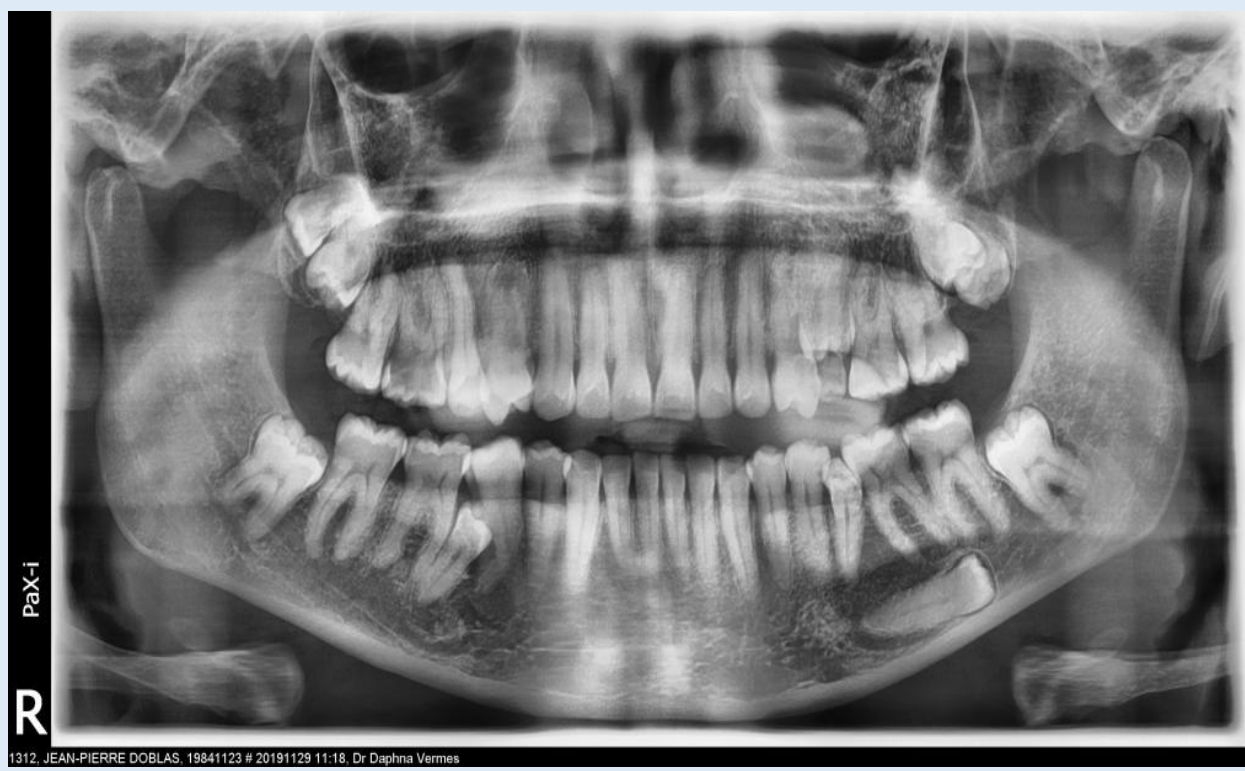

Figure 5: A panelipse radiograph of the jaws. Supplemental premolars in front of the lower first molars.

\section{Therapeutic Interventions}

Analgesics, desensitization of exposed dentine, conservative restoration of decayed teeth, or exodontia of the supplemental and impacted teeth were considered. The first three aforementioned were ruled out as they would produce no benefit to the patient in this case. For his immediate relief, focus was on the decayed offending premolar tooth which was causing 
pain, was out of alignment of the upper arch, had exposed dentin and a small area of decay. This was not worth retaining and was extracted under local analgesia. Also advised was selective removal of the other supplemental premolar-teeth outside the natural flow of the curves of the dental arches. The wisdom teeth and the supplemental upper third molars were all impacted, and surgical removal was recommended. The lower left horizontally impacted premolar was advised not to be removed, unless it caused symptoms.

\section{Discussion}

The case described manifests typical features of polydentulism. The patient illustrated here, had no other external malformation manifestations, other than extra eight supplemental teeth. A syndrome called "Multiple embedded or impacted teeth (no syndrome)" has been described [6]. This has been ascribed to an autosomal dominant trait, (the transmission of non-syndromal multiple supernumerary teeth [13-16]. Therapy usually indicates removal of impacted teeth because of danger of jaw fractures (especially the mandible) odontogenic infections, development of follicular cysts and rarely odontogenic neoplasia [6]. With stable jaw function, excellent oral hygiene and no noticeable symptoms, regular clinical and radiographic monitoring for growth or radiographic changes is advised. Lizards, many fish, sharks and dinosaurs are monomorphodont, that is they have teeth all the same shape. Also sharks are polyphyodont, as they have succedaneous generations of tooth replacement when teeth are lost or shed as they age. Some pundits aver that humans have three sets of teeth: besides the deciduous and permanent set, consideration needs to be focused on the iatrogenic implantprosthesis set [1-3]. Availability of qualified professional services, a dental clinic offering comprehensive up-to-date dentistry with a fully tooled-up operatory, mechanical processing and laboratories, adequate finance and/or insurance coverage with the right patient's attitude, approach knowledge and co-operation, are all necessary pre-requisites to be a reality, before a patient can successfully secure a third man-made set of functioning teeth. "Spectacular dentistry demands and commands spectacular prices", but the third set of teeth for those who have lost their dentition is available for those who can afford it $[17,18]$. In the case described here, all the supplementary teeth were well developed, with identifiable crowns and roots and none showed any concrescence or germination. Every natural human tooth has a unique anatomy which allows for identification of type and location where it was placed in the jaws. This feature is used extensively in paleodontology and archeology to interpret and classify evolution of mankind.

\section{Strengths and Limitations}

This case was recorded in a private office and presented unexpected abnormalities. Most qualified dental practitioners would immediately recognize aberrations, and although rare it is

Touyz LZG | Volume 1; Issue 2 (2020) | JDHOR-1(2)-009 | Case Report

Citation: Touyz LZG. Multiple Supplemental and Impacted Teeth (Polydent): A Non-Syndromic Case

Report. J Dental Health Oral Res. 2020;1(2):1-8.

DOI: http://dx.doi.org/10.46889/JDHOR.2020.1204 
important to recognize these cases, and provide sound targeted therapy and advice. Consequently, for this case a comprehensive cardiovascular, neurological, renal, dermatological and genetic work-up, while being highly desirable for academic purposes, these were not indicated or done. The patient never returned for follow-up treatment. The case report as presented provides adequate relevant findings, is informative and clinically significant.

This case had numerous supplemental teeth, no conical supernumeraries, but did show multiple impactions. Multiple supernumerary teeth are associated with cleidocranial dysplasia and Gardner syndrome. It is a rarity to encounter supernumeraries in people without some related pathology or other ectodermal disorder [6]. Among the most prevalent dental abnormalities (with increase or decrease in number of teeth) are people with genetic diseases affecting ectodermal structure like Cleidocranial Dysostosis (CCD: sometimes call Cleidocranail Dysplasia hypohydrotic ectodermal dysplasia, focal dermal hypoplasia, craniofacial dysostosis, and aperts syndrome [7]. Most commonly supplemental impacted teeth are found in those suffering from Cleidocranial Dysostosis (CCD). This (CCD) is an autosomal dominant trait, characterized by uni- or bilateral clavicle hypolplasia, skull abnormalities (from delayed closure of fontanelles), enlarged cranium with frontal bossing, a broad flat nose, exophthalmos, compromised hearing, and delayed eruption or multiple impactions of supplemental teeth [6,7].

\section{Conclusion and Significance}

This article clarifies what a supernumerary tooth is and differentiates between germination, concrescence and what a supplemental tooth is. Whenever an extra tooth is found on examination, many oral health care workers do not comprehend how to classify it; this understanding impacts on treatment. Therapy in this case was to resort to exodontias of the impacted molars and removal of the offending premolar-tooth outside the natural flow of the curves of the dental arches. This allows the residual erupted teeth within the arches to serve successfully as masticators. The lower left horizontally impacted premolar was not removed, should be monitored for any pathological changes, and only be removed if symptomatic. This case report presented here will assist researchers and oral health care workers to recognize and differentiate between supplemental teeth, supernumerary and impacted teeth. In the absence of other ectodermal or developmental anomalies, the final diagnosis was "Polydent: Multiple supplemental and impacted teeth (no syndrome)".

\section{References}

1. Profitt WR, Fields HW. In: contemporary orthodontics. Early stages of development. 1993;3:57-86.

2. Profitt WR, Fields HW. In: contemporary orthodontics. Later stages of development. 1993;4:87-104.

3. Grobstein C. Inductive tissue inter-actions in development. Amer Cancer Res. 1956;4:187-91.

4. Ruch JV. Tooth Morphogensis and differentiation. In: dentin and dentinogenesis. CRC Press. 1984;3:47-79.

Touyz LZG | Volume 1; Issue 2 (2020) | JDHOR-1(2)-009 | Case Report

Citation: Touyz LZG. Multiple Supplemental and Impacted Teeth (Polydent): A Non-Syndromic Case Report. J Dental Health Oral Res. 2020;1(2):1-8. 
5. Profitt WR, Fields HW. In: contemporary orthodontics. Problems involving crowding and malalignment. 1983;7:197-200.

6. Wood NK, Goaz PW. In: differential diagnosis of oral Lesion. Part III; Section C. 1980;28:591-2.

7. Laskaris G. In: color atlas of oral pathology. Genetic Diseases. 1988;4:32-33.

8. Neville BW, Damm DD, White DK. In: color atlas of clinical oral pathology. Cleidocranial Dysostosis. 1999;2:337-8.

9. Pindborg JJ. In: pathology od the dental hard tissues. Abnormalities of tooth morphology. 1970;1:27.

10. Cawson RA, Odell EW. In: essentials of oral pathology and oral medicine, disorders of development of teeth and related tissue. 1998;2:16-20.

11. Touyz LZG. The inverted maxillary premolar. 1979;34:377-9.

12. Batra P, Duggal R, Parkash H. Non-syndromic multiple supernumerary teeth transmitted as an autosomal dominant trait. J Oral Pathol Med. 2005;34(10):621-5.

13. Pindborg JJ. In: Pathology of the dental hard tissues. Abnormalities of tooth morphology. Morphological abnormalities. 1970;1:53-67.

14. Kaya GS, Yapici G, Omezli MM, Dayi E. Non-syndromic supernumerary premolars. Med Oral Patol Oral Cir Bucal. 2011;16(4):522-25.

15. Mali S, Karjodkar FR, Sontakke S, Sansare K. Supernumerary teeth in non-syndromic patients. Imaging Sci Dent. 2012;42(1):41-5.

16. Khambete N, Kumar R. Genetics and presence of non-syndromic supernumerary teeth: A mystery case report and review of literature. Contemp Clin Dent. 2012;3(4):499-502.

17. Touyz LZG. Sanative dentistry: Bringing sanity back into dentistry. Scientific Arch Dental Sci. 2018:115-19.

18. Touyz LZG. Stress and Coping strategies in Dentistry. J Depression and Anxiety. 2015;3:3. 